\title{
Longitudinal Assessment of Sleep Trajectories during Early Childhood and Their Association with Obesity
}

\author{
jill L. Kaar, PhD,' Sarah J. Schmiege, PhD, ' Heidi J. Kalkwarf, PhD, 3,4 \\ Jessica G. Woo, PhD, ${ }^{4,5}$ Stephen R. Daniels, MD, PhD,' and Stacey L. Simon, PhD'
}

\section{Abstract}

Introduction: To identify longitudinal sleep trajectories in early childhood and examine the influence of sleep duration on obesity risk via BMI percentile (BMIp).

Methods: Sleep, physical activity, and television viewing were measured in a cohort of 301 children, starting in 2001-2002, when children were 3 years and followed them through age 6. Nighttime sleep, daytime naps, and sleep duration were calculated. A series of latent growth curve models were used to estimate predictors of rate of change in sleep duration and BMIp overtime. A parallel process latent growth model examined the longitudinal relationship between sleep duration and BMIp simultaneously.

Results: Most children ( $>80 \%$ ) slept $>10$ hours per night across ages 3 to 6 years, despite the majority of children $(>66 \%)$ having bedtimes after $9 \mathrm{pm}$. Sleep duration decreased on average by 0.22 (95\% CI $0.20-0.24)$ hours each year of age $(p<0.001)$, while BMIp increased on average by $1.76(95 \%$ CI $1.18-2.34)$ each year of age $(p<0.001)$. Baseline sleep duration predicted the BMIp slope factor, over and above strong effects of baseline BMIp. This indicated that greater sleep duration at baseline was predictive of decreased BMIp over time (unstandardized coefficient $=-1.52(95 \%$ CI $0.34-2.71$, $p=0.012$ ).

Conclusions: Longer sleep duration at age 3 predicted decreased BMIp from ages 3 to 6 . These findings indicate that focusing on sleep behaviors in children before age 3 may be a priority for pediatric providers with a goal of decreasing obesity risk.

Keywords: longitudinal; preschoolers; sleep

\section{Introduction}

$\mathcal{C}$ hronic short sleep is a pervasive issue at all ages. The National Sleep Foundation reported that amount recommended, leading to a regular state of sleep restriction. ${ }^{1}$ Young children are at particular risk, as sleep problems are one of the most common parental complaints to pediatricians, ${ }^{2}$ and young children are more likely to be diagnosed with a sleep disorder than any other age group. ${ }^{3}$ Poor sleep in childhood tends to persist into adolescence and adulthood, developing into chronic sleep problems. ${ }^{4,5}$

Prior research has shown a relationship between poor sleep and obesity in children as young as 3 years. ${ }^{6-9}$ Short sleep duration has been shown to influence several important metabolic hormones, including leptin, ghrelin, insulin, cortisol, and growth hormone. ${ }^{10-16}$ Short sleep duration may also decrease metabolic rate (i.e., resting energy expenditure) and affect metabolic regulation. ${ }^{17-19}$ Insufficient sleep is thought to shift metabolism toward utilization of protein rather than fat for

\footnotetext{
'Department of Pediatrics, University of Colorado Anschutz Medical Campus, Children's Hospital Colorado, Aurora, CO.

${ }^{2}$ Department of Biostatistics and Informatics, Colorado School of Public Health, University of Colorado Anschutz Medical Campus, Aurora, CO.

${ }^{3}$ Division of Gastroenterology, Hepatology and Nutrition, Cincinnati Children's Hospital Medical Center, Cincinnati, OH.

${ }^{4}$ Department of Pediatrics, University of Cincinnati College of Medicine, Cincinnati, $\mathrm{OH}$.

${ }^{5}$ Division of Biostatistics and Epidemiology, Cincinnati Children's Hospital Medical Center, Cincinnati, $\mathrm{OH}$.
} 
energy generation, processes that are associated with insulin resistance and metabolic diseases, including type 2 diabetes and obesity.

In a cross-sectional study of 7-year olds, short sleep duration (less than 9 hours/night) was associated with increased risk of obesity, and these findings were independent of television (TV) watching or accelerometrymeasured time spent in sedentary activities. ${ }^{20}$ In a cohort of $>300$ children $4-10$ years old, obese children slept less than nonobese children and showed more variability in their sleep on the weekends, compared with school days. ${ }^{21}$ However, the existing studies are limited by use of retrospective parented-reported sleep and crosssectional designs, and few have investigated other key obesity-related health behaviors such as eating and activity. ${ }^{20-24}$

Of the few longitudinal studies conducted, some studies report shorter sleep duration in early childhood associated with increased weight gain and percent fat mass during later childhood, while others report no association. ${ }^{25-27}$ In a birth cohort from New Zealand $(n=244)$, sleep and other health behaviors were measured from age 3 to 7 years, and short sleep duration at ages 3-5 years was found to predict higher adiposity (measure via DEXA) at age $7 .{ }^{27}$ However, in a similar study conducted in the United States with more than 800 Hispanic children ages 4-19 years, parent-reported sleep duration did not predict increased weight gain 1 year later. ${ }^{25}$ These mixed results warrant the need for additional longitudinal studies to examine the stability of sleep during early childhood to identify optimal times to intervene to prevent the negative consequences of insufficient sleep, such as childhood obesity. The objective of this analysis was to (1) identify sleep trajectories in early childhood and (2) examine the influence of these trajectories on obesity risk. We hypothesized that shorter sleep duration during early childhood would negatively impact a child's obesity risk.

\section{Methods}

\section{Participants}

Participants in this analysis were children from a 20012002 prospective cohort study of healthy 3-year-old children from Cincinnati, $\mathrm{OH}$. Further details regarding this study have been previously published. ${ }^{28-35}$ Participants attended study visits every 4 months for 4 years during 2001-2006. A total of 372 children enrolled, and the current analysis included 301 children with complete data from ages 3 to 6 years. The 71 children excluded from this analysis did not differ from our analytical cohort $(n=301)$ based on socioeconomic characteristics. The study was approved by the Cincinnati Children's Hospital Medical Center Institutional Review Board and all parents/guardians provided written informed consent. The current analysis was conducted in 2019.

\section{Study Variables}

Study visits occurred every 4 months from when the children were recruited at age 3 through 6 years for each of the 301 children included in this analysis. Data from each of the visits were aggregated by study year and the individuals' average values for sleep, physical activity, and TV viewing were used in analysis. ${ }^{36}$ A triaxial actigraph (RT3; Stayhealthy, Inc., Monrovia, CA), worn on the hip, was used to assess physical activity and was worn for 3 days, including 2 weekdays and 1 weekend day. ${ }^{31}$ Minutes of moderate/vigorous physical activity (MVPA) were calculated by the number of minutes $>1400$ counts. During the same days as the actigraph was worn, parents were asked to complete an activity diary in which the child's sleep for each of the three nights was recorded as the time the child went to bed and the time the child woke the following day. Nighttime sleep duration, daytime nap duration, and 24-hour sleep duration (nighttime sleep plus daytime naps) were calculated using the average of all 3 days.

Parents were also asked during these 3 days "how much time did your child spend watching TV or videotapes?" which was used to estimate average hours per day spent watching TV. Demographic data were collected via questionnaires completed by parents and included participant age, sex, race, and household income. Height and weight were measured during the research visit using a Health-O-Meter (Alsip, IL) electronic scale and a custom stadiometer. BMI was calculated and ageand sex-specific BMI percentiles (BMIps) were derived

\begin{tabular}{|c|c|}
\hline & Frequency (\%) \\
\hline \multicolumn{2}{|l|}{ Sex } \\
\hline Boys & $153(51)$ \\
\hline Girls & 148 (49) \\
\hline \multicolumn{2}{|l|}{ Race } \\
\hline White & $248(82)$ \\
\hline Black & $53(18)$ \\
\hline \multicolumn{2}{|l|}{ Annual household income } \\
\hline Low income $(<\$ 50,000)$ & $110(36)$ \\
\hline \multicolumn{2}{|l|}{ Obesity status @ 3 years of age } \\
\hline Obese ( $\geq 95$ th percentile) & $23(8)$ \\
\hline Overweight ( $\geq 85-94$ th percentile) & $39(13)$ \\
\hline \multicolumn{2}{|l|}{ Obesity status @6 years of age } \\
\hline Obese ( $\geq 95$ th percentile) & $38(13)$ \\
\hline Overweight ( $\geq 85-94$ th percentile) & $56(19)$ \\
\hline
\end{tabular}




\begin{tabular}{|c|c|c|c|c|}
\hline & Age 3 & Age 4 & Age 5 & Age 6 \\
\hline \multicolumn{5}{|l|}{ All $(n=30$ I $)$} \\
\hline BMI percentile (\%) & $61.1 \pm 27.0$ & $63.1 \pm 26.7$ & $65.1 \pm 26.7$ & $66.6 \pm 26.2$ \\
\hline Total sleep duration ${ }^{\mathrm{a}}$ (hours) & $11.2(9.7-13.4)$ & $10.7(8.9-12.9)$ & $10.7(8.1-12.7)$ & $10.5(8.3-12.6)$ \\
\hline Daytime naps (minutes) & $40.0(26.2-52.5)$ & $30.0(20.0-45.0)$ & $35.0(20.0-60.0)$ & $0(0-0)$ \\
\hline Nighttime sleep duration (hours) & $10.6(8.7-12.6)$ & $10.7(8.2-12.9)$ & $10.6(8.1-12.7)$ & $10.5(8.3-12.6)$ \\
\hline Bedtime & $2 I: 3 \mid(|8: 47-0|: 25)$ & $21: 27(\mid 9: 31-0: 48)$ & $21: 21 \quad(19: 24-0: 19)$ & $21: 26(19: 53-0: 28)$ \\
\hline Wake time & $8: 07(6: 08-12: 10)$ & 8:07 (5:38-|I:23) & $7: 58(6: 01-|0: 3|)$ & $7: 52(6: 07-10: 43)$ \\
\hline \multicolumn{5}{|l|}{ Boys $(n=153)$} \\
\hline BMI percentile (\%) & $60.5 \pm 26.9$ & $62.4 \pm 26.6$ & $64.5 \pm 26.7$ & $66.4 \pm 26.2$ \\
\hline Total sleep duration ${ }^{\mathrm{a}}$ (hours) & $11.2(9.7-13.3)$ & $10.7(8.9-12.9)$ & $10.7(8.8-12.7)$ & $10.5(8.8-12.6)$ \\
\hline Daytime naps (minutes) & $38.3(25.0-51.7)$ & $28.3(18.3-40.0)$ & $37.5(22.5-50.0)$ & $0(0-0)$ \\
\hline Nighttime sleep duration (hours) & $10.6(8.7-12.6)$ & $10.7(8.9-12.9)$ & $10.7(8.8-12.7)$ & $10.5(8.8-12.6)$ \\
\hline Bedtime & $21: 29(|8: 47-0|: 25)$ & $21: 26(|9: 3|-0: 48)$ & $21: 19(19: 25-23: 34)$ & $21: 25(19: 53-0: 01)$ \\
\hline Wake time & $8: 08(6: 08-12: 10)$ & $8: 05(6: 25-11: 19)$ & $7: 58(6: 30-10: 07)$ & $7: 52(6: 11-10: 01)$ \\
\hline \multicolumn{5}{|l|}{ Girls $(n=148)$} \\
\hline BMI percentile (\%) & $61.6 \pm 27.2$ & $63.8 \pm 27.0$ & $65.8 \pm 26.7$ & $66.7 \pm 26.3$ \\
\hline Total sleep duration ${ }^{\mathrm{a}}$ (hours) & $11.2(9.7-13.4)$ & $10.8(9.0-12.9)$ & $10.6(8.1-12.5)$ & $10.4(8.3-11.9)$ \\
\hline Daytime naps (minutes) & $42.5(26.7-53.3)$ & $37.1(20.0-47.5)$ & $35.0(20.0-60.0)$ & $0(0-0)$ \\
\hline Nighttime sleep duration (hours) & $10.5(8.8-12.6)$ & $10.7(8.2-12.9)$ & $10.6(8.1-12.5)$ & $10.4(8.3-11.9)$ \\
\hline Bedtime & $21: 32(19: 45-23: 40)$ & $21: 28(19: 33-0: 15)$ & $21: 25$ (19:59-0:19) & $21: 28(20: 00-24: 28)$ \\
\hline Wake time & $8: 04(6: 16-10: 35)$ & $8: 08(5: 38-11: 23)$ & $7: 58(6: 01-10: 30)$ & $7: 53(6: 07-10: 43)$ \\
\hline
\end{tabular}

Minutes of daytime naps are summarized using median (IQR). Data include mean $\pm S D$, mean (min-max), or median (IQR).

${ }^{a}$ Total sleep duration includes nighttime sleep duration plus daytime nap duration.

$\mathrm{IQR}$, interquartile range; SD, standard deviation.

using the Centers for Disease Control and Prevention growth charts.

\section{Statistical Analysis}

Analyses were conducted in SAS 9.4 (SAS Institute, Cary, NC) and Mplus v8. A series of latent growth curve models were estimated to test the average rate of change in sleep duration and BMIp over time, variability in change, and predictors of the initial status factor (age 3) and the slope factor (change over time). First, separate unconditional models were estimated for both sleep duration and BMIp to examine trajectories over time in the absence of other explanatory variables. Next, conditional models were estimated to test child gender, race, MVPA, and TV viewing as potential predictors of baseline values and change over time of sleep duration and BMIp. As a final step in the model building process, we estimated a parallel process latent growth model to examine the longitudinal relationship between sleep duration and BMIp. The growth factors (e.g., initial status and slope) of each variable were simultaneously modeled to test associations between sleep duration and BMIp at baseline and over time. Of primary interest was the relationship of baseline (age 3) sleep duration with baseline BMIp (age 3) and BMIp over time (slope), although associations among all four growth parameters were estimated for completeness. This final model adjusted for child gender, race, TV viewing, and MVPA (although primary conclusions were unchanged between the adjusted and unadjusted models).

\section{Results}

The analytical cohort was $49 \%$ female, predominately white $(82 \%)$ with $36 \%$ having household incomes below $\$ 50,000$ (Table 1). At age 3, 21\% were 

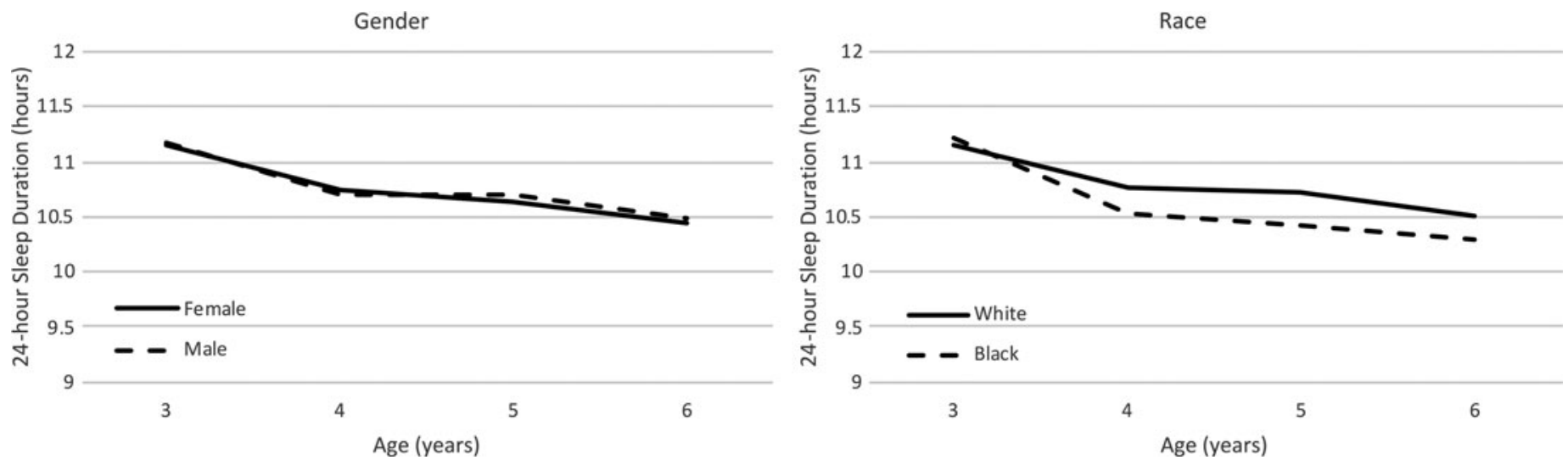

Figure 1. Changes in total sleep duration of time by child gender and race.

defined as overweight or obese and at age 6, 32\% were defined as overweight or obese. Children had a similar bed and wake time across ages 3 to 6 years with the majority of children reported as having a bedtime after 9 pm (Table 2). Most children took naps at age 3 (94\%), but by age 6 years, none of the children in this cohort was taking naps.

The latent growth curve model examining change in sleep duration showed a small but significant decrease over time (Table 2). At baseline, the sleep duration average was 11.2 hours and decreased on average by 0.22 (95\% CI $0.20-0.24)$ hours each year of age $(p<0.001)$. Child gender did not predict baseline sleep or change in sleep over time (Fig. 1); however, children categorized as black had significantly steeper decline of sleep duration from ages 3 to 6 years compared with children categorized as white [0.08 hours steeper decrease per year of age $(95 \% \mathrm{CI}$ $0.02-0.14)]$. Sleep duration (at baseline or over time) was also not influenced by MVPA or TV viewing (all $p \mathrm{~s}>0.24)$.

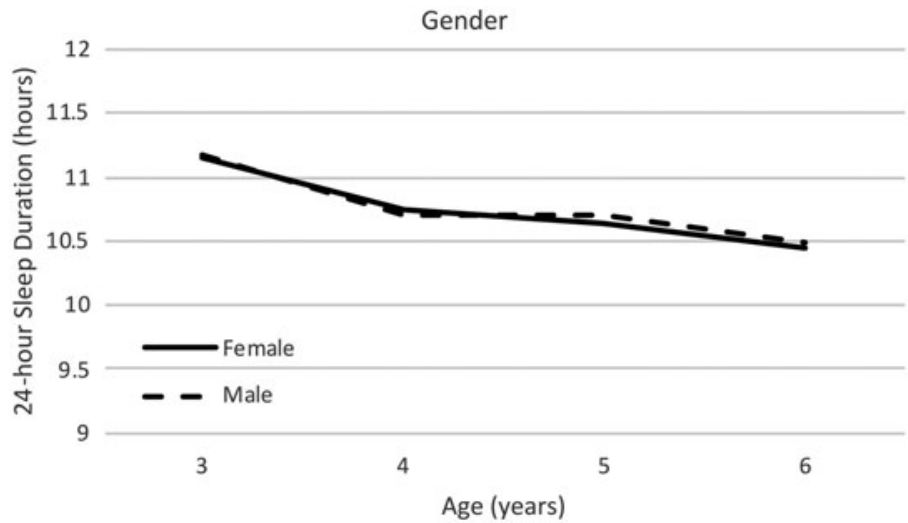

BMIp demonstrated a significant increase over time. At baseline, average BMIp was 61.1 (Table 2) and increased on average by 1.76 (95\% CI 1.18-2.34) each year of age $(p<0.001)$. Neither baseline BMIp nor changes in BMIp over time were influenced by child gender or race (Fig. 2; all $p \mathrm{~s}>0.29)$. TV viewing was associated with higher BMIp at baseline [0.048 increase (95\% CI 0.005-0.09) for every unit increase in TV viewing $(p=0.03)$ but not with changes in BMIp over time $(p=0.82)]$. MVPA was unrelated to BMIp at baseline or over time (both $p \mathrm{~s}>0.33$ ).

Figure 3 displays the results of the parallel process growth model simultaneously testing longitudinal relationships of sleep duration with BMIp. Standardized beta coefficients are reported so that all relationships may be examined on a common scale. Baseline (age 3) sleep duration was unrelated to baseline BMIp $(p=0.36)$, and change in sleep duration was unrelated to change in BMIp $(p=0.24)$. Consistent with study hypotheses, baseline sleep duration predicted the BMIp

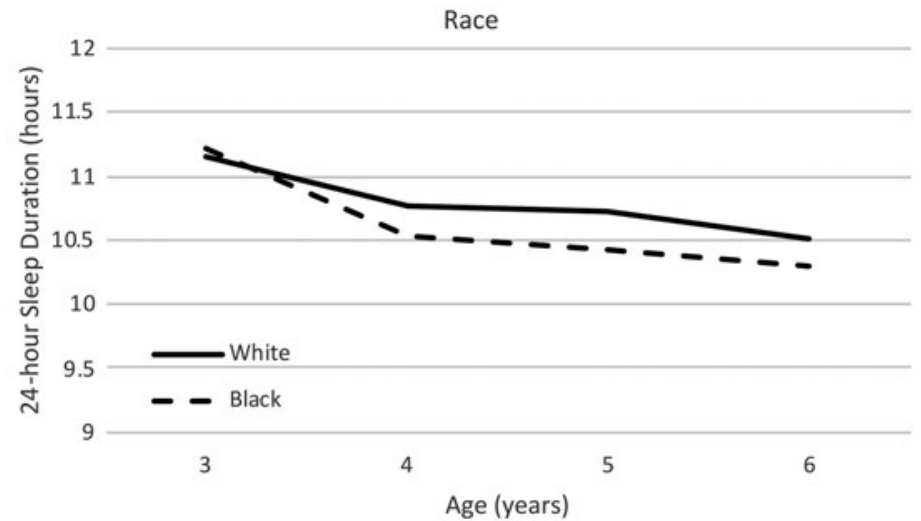

Figure 2. Changes in BMI percentile over time by child gender and race. 


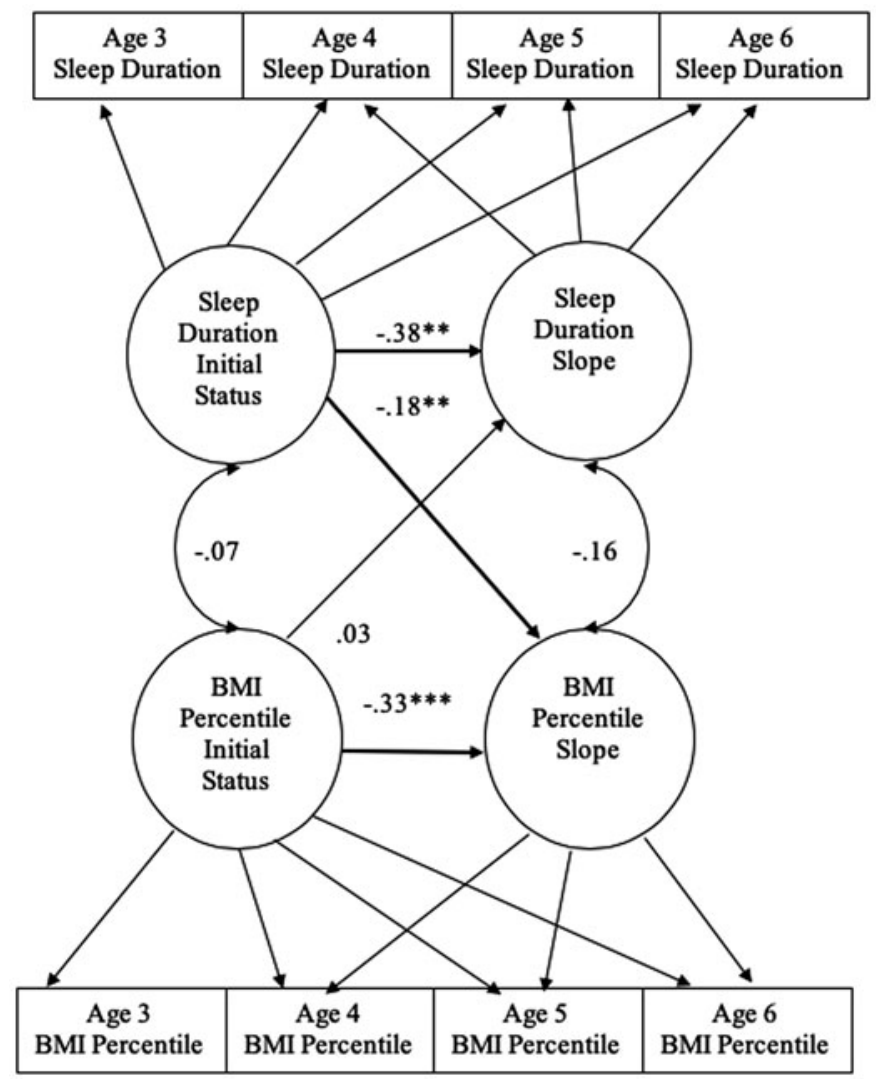

Covariates:

- Child Gender

- Child Race

- Television viewing (hours/day)

- Moderate-Vigorous Physical Activity (minutes/day)

Figure 3. Parallel process latent growth curve model showing longitudinal relationships between sleep duration and BMI percentile. Note, reported values are standardized beta coefficients. $* * p<0.01 ; * * * p<0.001$.

slope factor, over and above strong effects of baseline BMIp. This indicated that greater sleep duration at baseline was predictive of decreased BMIp over time (unstandardized coefficient $=-1.52(95 \%$ CI $0.34-2.71$, $p=0.012$ ).

\section{Discussion}

In our cohort of 301 children followed from age 3 to 6 years, we found that sleep duration significantly decreased over time by an average of 0.22 hours each year of age (i.e., 13 minutes). A child's baseline sleep duration (age 3) was predictive of BMIp changes over time, suggesting that children with higher sleep duration had a trajectory of lower BMIp over time. The children in our cohort tended to meet the minimum recommended hours of sleep for children ages $3-5$ years ( $>10$ hours/day), with only $5 \%-19 \%$ reported as having insufficient sleep at any given age.
Although we did not see any significant differences in this decline by child gender, we did find that children categorized as the black race had a significantly steeper decline in sleep compared with children categorized as the white race.

The finding that the majority of children achieved sufficient sleep is similar to another study conducted in a white, middle class population where $87 \%$ of children ages 1-8 years met aged-based recommendations for sleep. ${ }^{37}$ However, in a sample of low-income, African American and Hispanic children ages 3-5 years, over half $(61.9 \%)$ were categorized with insufficient sleep $\left(<10\right.$ hours/day). ${ }^{38}$ Similarly, we found that the black race, compared with white, was a significant predictor of decreased sleep over time in our cohort.

Our findings should be interpreted with caution as they may not be representative of all young children. The cohort was not recruited with the intent to be nationally or locally representative, and due to the location of study enrollment only white and black children but no other racial or ethnic groups were enrolled. As this was a secondary analysis from a larger, longitudinal study, it was not originally designed to address the specific research questions presented in this article. Future studies should calculate a priori an adequate sample size to have sufficient statistical power to observe such an effect. In addition, the study did use rigorous methods for physical activity by using accelerometry; however, sleep variables were collected via parent report, which may be subject to bias. ${ }^{39,40}$ Strengths of our study include following a large number of children over 4 years with multiple measures of key health behaviors. Future work should be conducted to replicate our findings in a more diverse, longitudinal cohort with objective measures of sleep duration and timing.

In conclusion, we observed that longer sleep duration at age 3 predicted decreased BMIp from ages 3 to 6 years. Pediatric providers should consider focusing on sleep behaviors with their patients at a young age. Based on our findings, future interventions should be explored focusing on sleep behaviors before age 3 as a means of improving sleep and reducing obesity risk in children.

\section{Funding Information}

Funded by the American Heart Association Scientist Development Award (16SDG29170007; PI: J.L.K.) and the National Heart, Lung, \& Blood Institute, National Institute of Health (R01 HL064022).

\section{Author Disclosure Statement}

No competing financial interests exist. 


\section{References}

1. National Sleep Foundation. Lack of Sleep is Affecting Americans, Finds the National Sleep Foundation, December 2014. www .sleepfoundation.org/shi (Last accessed November 14, 2019).

2. Armstrong KL, Quinn RA, Dadds MR. The sleep patterns of normal children. Med J Aust 1994;161:202-206.

3. Meltzer LJ, Johnson C, Crosette J, et al. Prevalence of diagnosed sleep disorders in pediatric primary care practices. Pediatrics 2010;125:e1410-e1418.

4. Beebe DW, Rausch J, Byars KC, et al. Persistent snoring in preschool children: Predictors and behavioral and developmental correlates. Pediatrics 2012;130:382-389.

5. Byars KC, Yolton K, Rausch J, et al. Prevalence, patterns, and persistence of sleep problems in the first 3 years of life. Pediatrics 2012;129:e276-e284.

6. Taveras EM, Rifas-Shiman SL, Oken E, et al. Short sleep duration in infancy and risk of childhood overweight. Arch Pediatr Adolesc Med 2008;162:305-311.

7. Bell JF, Zimmerman FJ. Shortened nighttime sleep duration in early life and subsequent childhood obesity. Arch Pediatr Adolesc Med 2010;164:840-845.

8. Cappuccio FP, Taggart FM, Kandala NB, et al. Meta-analysis of short sleep duration and obesity in children and adults. Sleep 2008; 31:619-626.

9. Landhuis CE, Poulton R, Welch D, Hancox RJ. Childhood sleep time and long-term risk for obesity: A 32-year prospective birth cohort study. Pediatrics 2008;122:955-960.

10. Beihl DA, Liese AD, Haffner SM. Sleep duration as a risk factor for incident type 2 diabetes in a multiethnic cohort. Ann Epidemiol 2009;19:351-357.

11. Berentzen NE, Smit HA, Bekkers MB, et al. Time in bed, sleep quality and associations with cardiometabolic markers in children: The prevention and incidence of asthma and mite allergy birth cohort study. J Sleep Res 2014;23:3-12.

12. Buxton OM, Pavlova M, Reid EW, et al. Sleep restriction for 1 week reduces insulin sensitivity in healthy men. Diabetes 2010;59: 2126-2133.

13. Cappuccio FP, D'Elia L, Strazzullo P, Miller MA. Quantity and quality of sleep and incidence of type 2 diabetes: A systematic review and meta-analysis. Diabetes Care 2010;33:414-420.

14. Cedernaes J, Schioth HB, Benedict C. Determinants of shortened, disrupted, and mistimed sleep and associated metabolic health consequences in healthy humans. Diabetes 2015;64: 1073-1080.

15. Klingenberg L, Chaput JP, Holmback U, et al. Acute sleep restriction reduces insulin sensitivity in adolescent boys. Sleep 2013; 36:1085-1090.

16. Knutson KL, Van Cauter E. Associations between sleep loss and increased risk of obesity and diabetes. Ann N Y Acad Sci 2008; 1129:287-304.

17. Koren D, O’Sullivan KL, Mokhlesi B. Metabolic and glycemic sequelae of sleep disturbances in children and adults. Curr Diab Rep 2015;15:562.

18. Reutrakul S, Van Cauter E. Interactions between sleep, circadian function, and glucose metabolism: Implications for risk and severity of diabetes. Ann N Y Acad Sci 2014;1311:151173.
19. Spiegel K, Tasali E, Penev P, Van Cauter E. Brief communication: Sleep curtailment in healthy young men is associated with decreased leptin levels, elevated ghrelin levels, and increased hunger and appetite. Ann Intern Med 2004;141:846-850.

20. Nixon GM, Thompson JM, Han DY, et al. Short sleep duration in middle childhood: Risk factors and consequences. Sleep 2008;31: $71-78$

21. Spruyt K, Molfese DL, Gozal D. Sleep duration, sleep regularity, body weight, and metabolic homeostasis in school-aged children. Pediatrics 2011;127:e345-e352.

22. Chaput JP, Gray CE, Poitras VJ, et al. Systematic review of the relationships between sleep duration and health indicators in school-aged children and youth. Appl Physiol Nutr Metab 2016; 41(6 Suppl 3):S266-S282.

23. Chaput JP, Lambert M, Gray-Donald K, et al. Short sleep duration is independently associated with overweight and obesity in Quebec children. Can J Public Health 2011;102:369-374.

24. Gupta NK, Mueller WH, Chan W, Meininger JC. Is obesity associated with poor sleep quality in adolescents? Am J Hum Biol 2002;14:762-768.

25. Butte NF, Cai G, Cole SA, et al. Metabolic and behavioral predictors of weight gain in Hispanic children: The Viva la Familia Study. Am J Clin Nutr 2007;85:1478-1485.

26. Hjorth MF, Chaput JP, Ritz C, et al. Fatness predicts decreased physical activity and increased sedentary time, but not vice versa: Support from a longitudinal study in 8- to 11-year-old children. Int $J$ Obes (2005) 2014;38:959-965.

27. Carter PJ, Taylor BJ, Williams SM, Taylor RW. Longitudinal analysis of sleep in relation to BMI and body fat in children: The FLAME study. BMJ 2011;342:d2712.

28. Burdette HL, Whitaker RC, Hall WC, Daniels SR. Maternal infantfeeding style and children's adiposity at 5 years of age. Arch Pediatr Adolesc Med 2006;160:513-520.

29. Burdette HL, Whitaker RC, Hall WC, Daniels SR. Breastfeeding, introduction of complementary foods, and adiposity at $5 \mathrm{y}$ of age. Am J Clin Nutr 2006;83:550-558.

30. Edwards NM, Kalkwarf HJ, Woo JG, et al. Child physical activity associations with cardiovascular risk factors differ by race. Pediatr Exerc Sci 2016;28:397-406.

31. Edwards NM, Khoury PR, Kalkwarf HJ, et al. Tracking of accelerometer-measured physical activity in early childhood. Pediatr Exerc Sci 2013;25:487-501.

32. Edwards NM, Myer GD, Kalkwarf HJ, et al. Outdoor temperature, precipitation, and wind speed affect physical activity levels in children: A longitudinal cohort study. J Phys Act Health 2015;12: 1074-1081.

33. Woo JG, Sucharew $\mathrm{H}, \mathrm{Su} \mathrm{W}$, et al. Infant weight and length growth trajectories modeled using superimposition by translation and rotation are differentially associated with body composition components at 3 and 7 years of age. J Pediatr 2018; 196:182-188.e181.

34. Wosje KS, Khoury PR, Claytor RP, et al. Dietary patterns associated with fat and bone mass in young children. Am J Clin Nutr 2010;92:294-303.

35. Wosje KS, Khoury PR, Claytor RP, et al. Adiposity and TV viewing are related to less bone accrual in young children. $J \mathrm{Pe}$ diatr 2009;154:79-85.e72.

36. Paruthi S, Brooks LJ, D'Ambrosio C, et al. Recommended amount of sleep for pediatric populations: A consensus statement of the 
American academy of sleep medicine. J Clin Sleep Med 2016;12: 785-786.

37. Plumptre L, Anderson LN, Chen Y, et al. Longitudinal analysis of sleep duration and cardiometabolic risk in young children. Child Obes 2017;13:291-299.

38. Vezina-Im LA, Hughes SO, Baranowski T, Nicklas TA. Association between sleep duration and body mass index among US low-income preschoolers. Obesity (Silver Spring) 2017;25: 1770-1775.

39. Galland BC, Taylor BJ, Elder DE, Herbison P. Normal sleep patterns in infants and children: A systematic review of observational studies. Sleep Med Rev 2012;16:213-222.
40. Iglowstein I, Jenni OG, Molinari L, Largo RH. Sleep duration from infancy to adolescence: Reference values and generational trends. Pediatrics 2003;111:302-307.

Address correspondence to: Jill L. Kaar, PhD

Children's Hospital Colorado 13123 East 16th Avenue, Box 265 Aurora, CO 80045

E-mail: jill.kaar@ucdenver.edu 